\title{
Title: Linking mechanistic analysis of catalytic reactivity cliffs to ligand classification
}

Authors: Samuel H. Newman-Stonebraker ${ }^{1 \dagger}$, Sleight R. Smith ${ }^{2 \dagger}$, Julia E. Borowski ${ }^{1}$, Ellyn Peters $^{2}$, Tobias Gensch ${ }^{2}$, Heather C. Johnson ${ }^{3}$, Matthew S. Sigman ${ }^{2 *}$, Abigail G. Doyle ${ }^{1 *}$

\section{Affiliations:}

${ }^{1}$ Department of Chemistry, Princeton University, Princeton, NJ 08544.

${ }^{2}$ Department of Chemistry, University of Utah, Salt Lake City, UT 84112.

${ }^{3}$ Process Research and Development, Merck \& Co., Inc., Rahway, NJ 07065

*Corresponding author. Email: sigman@chem.utah.edu (M.S.S.), agdoyle@princeton.edu (A.G.D.)

These authors contributed equally

Abstract: Statistical analysis of reaction data with molecular descriptors can enable chemists to identify reactivity cliffs that result from a mechanistic dependence on a specific structural feature. In this study, we develop a broadly applicable and quantitative classification workflow that identifies reactivity cliffs in eleven $\mathrm{Ni}$ - and Pd-catalyzed cross-coupling datasets employing monodentate phosphine ligands. A unique ligand steric descriptor, $\% V_{\text {bur }}(\min )$, is found to divide these datasets into active and inactive regions at a similar threshold value. Organometallic studies demonstrate that this threshold corresponds to the binary outcome of bisligated versus monoligated metal and that $\% V_{\text {bur }}(\min )$ is a physically meaningful and predictive representation of ligand structure in catalysis. Taken together, we expect that this strategy will be of broad value in mechanistic investigation of structure-reactivity relationships, while providing a means to rationally partition datasets for data-driven modeling.

\section{Main text:}

For almost 100 years, chemists have used data-driven modeling to understand the many complex relationships connecting chemical structure to reactivity. The paradigmatic example is the 
29 Hammett equation (1), a linear free energy relationship (LFER) between an experimental observable and a molecular descriptor (2). LFERs have been used extensively to understand reaction mechanism $(3,4)$ and, in certain cases, to predict reaction outcomes $(5)$. While many

32 reactions are continuously dependent on a single descriptor, as captured by LFERs, some processes may exhibit reactivity cliffs, wherein a criterion or threshold value of a given feature must be met

34 in order for the reaction to occur (i.e., a binary response) (6). If a particular molecular feature can define a reactivity cliff and classify molecules by structure, this could be used to predict reaction performance of unseen examples while also resulting in the development of key mechanistic

37 insights (Fig. 1A). Thus, the identification and understanding of these reactivity cliffs is of great interest, as the ability to bifurcate datasets - and the molecules within them-on the basis of 39 distinct mechanistic outcomes would augment expert chemists' intuition and simplify subsequent data-driven modeling.

Transition metal-catalyzed cross coupling represents an interesting case study for 42 identifying reactivity cliffs. This reaction class is synthetically important due to its widespread use 43 in the construction of pharmaceuticals (7) and materials (8). In addition, the success of these 44 reactions is highly dependent on ligand identity, for which monodentate phosphines are ubiquitous $45(9,10)$. As a result, chemists have developed numerous tools to quantitatively describe the diverse 46 steric and electronic properties of these ligands - for which thousands of unique structures exist47 to uncover structure-reactivity relationships (SRRs) in experimental data (11) (Fig. 1B). These 48 descriptors, including Tolman Cone Angle (12, 13), Solid Angle (14), Sterimol (15), and percent 49 buried volume $\left(\% V_{\text {bur }}\right)(16,17)$, have been used successfully in linear modeling of cross-coupling 50 reactions $(5,18)$ (Fig. 1C). However, there are situations in which seemingly similar ligands afford 
51 substantially different responses (19), suggesting the presence of discontinuous trends in ligand 52 activity (20).

Considering this, we hypothesized that reactivity cliffs were embedded in a phosphine 54 structural feature that had yet to be recognized. Identification of such a feature would not only 55 allow for the development of a statistical tool to classify active and inactive ligands but also 56 provide a quantitative handle for mechanistically rationalizing ligand performance. Herein we 57 provide a workflow and analysis to achieve these goals. Using the organophosphorus(III) 58 descriptor library recently developed by Sigman and Aspuru-Guzik (Kraken) that possesses over 59190 conformationally representative descriptors for each of several thousand monodentate 60 phosphines (21) (Fig. 1D), we were able to classify eleven Ni and Pd catalytic case studies into 61 mechanistically bifurcated regions of reactivity based on catalyst ligation state, consistent with 62 spectroscopic/crystallographic organometallic studies. We found that minimum percent buried 63 volume $\left(\% V_{\text {bur }}(\min )\right)$ — a previously unexplored descriptor that quantifies the proximal steric bulk 64 of the smallest energetically accessible conformation of a given ligand - is the single descriptor 65 able to achieve this classification. Ultimately, we demonstrate that this analysis was able to reveal 66 non-intuitive trends in organometallic chemistry and thereby serves as an important mechanistic 67 tool, complementary to LFERs $(18,19)$, to understand and predict monodentate phosphine SRRs 68 and catalyst speciation in cross-coupling catalysis (Fig. 1E). 

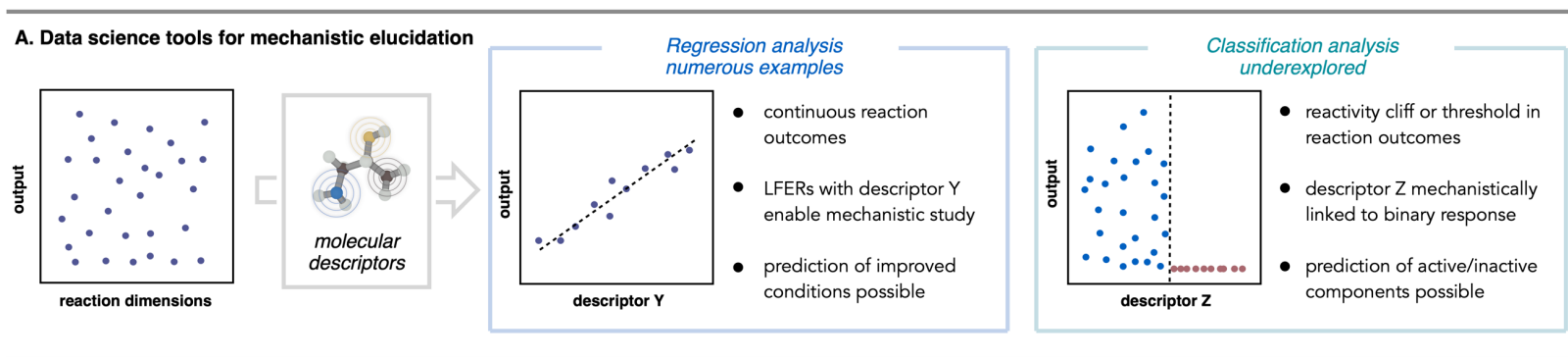

B. Phosphine ligand structural diversity
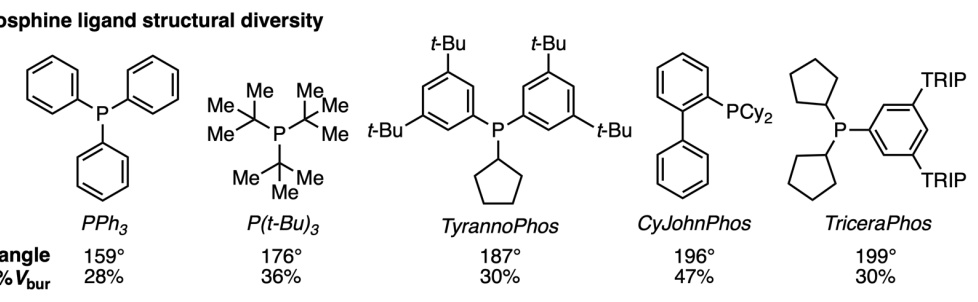

C. Traditional phosphine steric descriptors
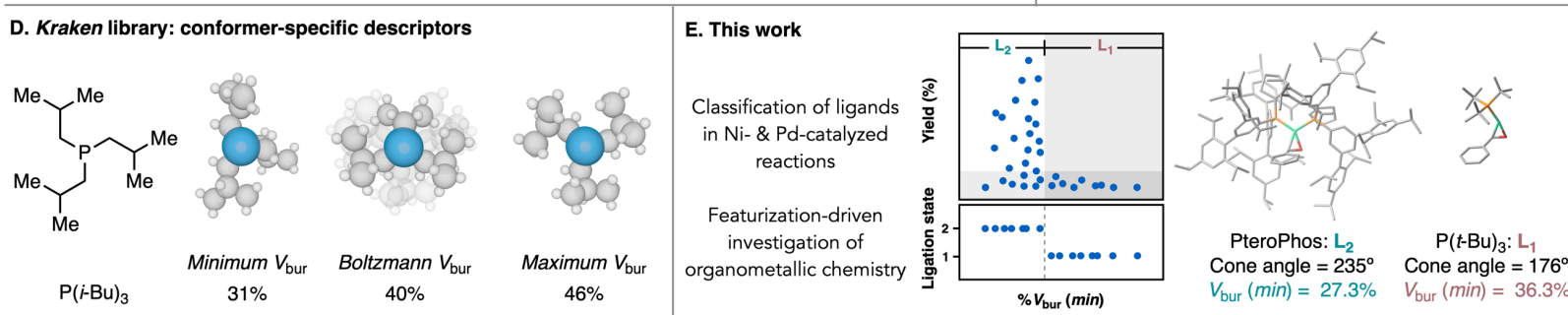

Fig. 1. Introduction. (A) Tools to understand trends in chemical reactivity using mechanistically relevant molecular descriptors. (B) Examples of monodentate phosphines used in $\mathrm{Ni}$ and $\mathrm{Pd}$ cross-coupling reactions, including TyrannoPhos and TriceraPhos (DinoPhos ligands) recently reported by the Doyle lab. TRIP $=$ 2,4,6-triisopropylphenyl. (C) Commonly employed methods of quantifying phosphine steric properties. (D) Phosphine descriptor library (Kraken) capturing multiple ligand conformers. (E) This work.

\section{Results and Discussion}

Exploration of phosphine steric features in Ni-catalysis.

Our initial platform for probing the presence of ligand reactivity cliffs was inspired by a recent study from the Doyle group that identified a new class of phosphine ligands - the DinoPhos ligands (TriceraPhos and TyrannoPhos (Fig. 1B)) - for Ni-catalyzed cross-coupling of acetals with aryl boroxines (19). Having both large cone angles and small $\% V_{\text {bur }}$ values, these ligands are distinct from other phosphines that often have a 1:1 relationship between these features (22). The large cone angles suggested that the DinoPhos ligands may behave like the sterically bulky 
84 monoligated $\mathrm{Pd}\left(\mathrm{L}_{1}\right.$ complexes $)$ has been shown to be critical for reaction success (23-27).

85 However, the small $\% V_{\text {bur }}$ characterized these ligands as similar to smaller structures (e.g., $\left.\mathrm{PPh}_{3}\right)$

86 that tend to form bisligating (denoted as $\mathrm{L}_{2}$ ) complexes. The observation that this unique steric

87 profile was essential for reaction success in Ni catalysis raised questions about its impact on 88 speciation and prompted us to use Ni-catalyzed cross-coupling reactions as a case study for the 89 identification of ligand reactivity cliffs.

9115 additional ligands to span the entire range of $\% V_{\text {bur }}$ (I, Figure $\left.2 \mathrm{~A}\right)$. We also collected data on 92 more traditional $\mathrm{Csp}^{2}-\mathrm{Csp}^{2}$ Suzuki-Miyaura coupling reactions with aryl chlorides using highthroughput experimentation (HTE) (28). The ligand set was selected from a combined Doyle and 94 Merck inventory to encompass 90 monodentate ligands that are evenly distributed throughout the feature space from the Sigman/Aspuru-Guzuk virtual library (21) as determined by principal component analysis. These ligands were evaluated for four cross-coupling reactions wherein the 97 aryl halide and aryl boronic acid were altered (II-V, Figure 2A). The coupling partners included a range of electronic and steric features that could impact different aspects of the elementary steps of the catalytic cycle. Notably, the DinoPhos ligands were top performers in each of these reactions, along with DrewPhos (29), a triarylphosphine ligand that possesses large 3,5substituents similar to the DinoPhos ligands. descriptors relate to reaction performance. For each reaction, the yield was evaluated against representative descriptors in the library; as three examples, the cone angle (30), Boltzmann averaged $\% V_{\text {bur }}$ of the ligand's conformational ensemble $\left(\% V_{\text {bur }}(\right.$ Boltz $\left.)\right)$, and $\% V_{\text {bur }}$ of the library 106 conformer with the smallest buried volume $\left(\% V_{\text {bur }}(\min )\right)$ are shown in Figure $2 \mathrm{~B}$. While the cone 
107 angle descriptors did not provide clear reactivity cutoffs, both $\% V_{\text {bur }}\left(\right.$ Boltz) and $\% V_{\text {bur }}(\min )$ 108 allowed for visual classification of ligand performance into "active" and "inactive" bins (details 109 of the classification tool will be described below), though several outliers were present with the 110 former. $\% V_{\text {bur }}(\mathrm{min})$ revealed sharp cutoffs in reactivity, wherein nearly all ligands above $32 \%$ 111 were found to be unreactive. Ligands that were unsuccessful at promoting reactivity below $32 \%$ $112 V_{\text {bur }}(\min )$ were grouped in their own bin, wherein electronic features and catalyst-poisoning 113 functionality (e.g., cyano, carbonyl, and halide groups) appeared to be largely responsible for their 114 inactivity. Notably, the use of $\% V_{\text {bur }}(\mathrm{min})$ but not $\% V_{\text {bur }}($ Boltz) allowed for various highly-flexible 115 scaffolds such as $\mathrm{P}(i-\mathrm{Bu})_{3}, \mathrm{PBn}_{3}$, and small Buchwald-type phosphines $(31,32)$, to be successfully 116 classified. Thus, this steric descriptor was best able to categorize phosphines into active and 117 inactive groups, with a reactivity threshold of $\sim 32 \% V_{\text {bur }}(\mathrm{min})$ appearing to be largely independent 118 of the substrate pairing. 


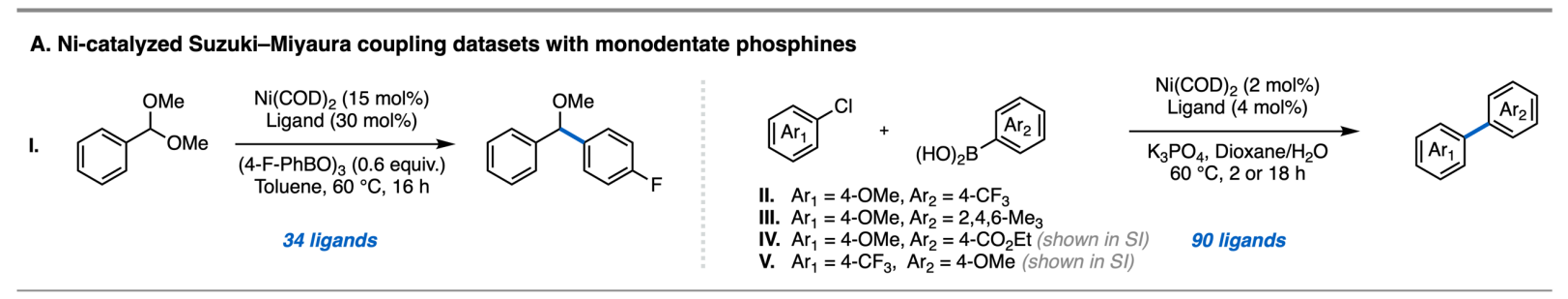

B. Univariate analysis of datasets with cone angle and \% $V_{\text {bur }}(B o / t z) /(m i n)$

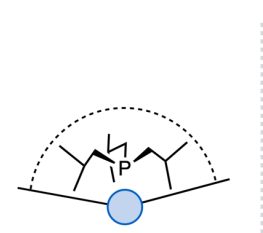

Cone angle

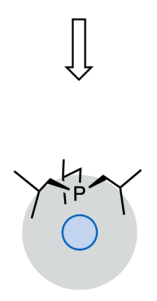

$\% V_{\text {bur }}($ Boltz)<smiles>C=CC=C</smiles>

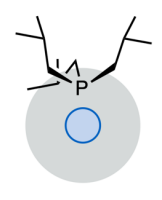

$\% V_{\text {bur }}(\min )$
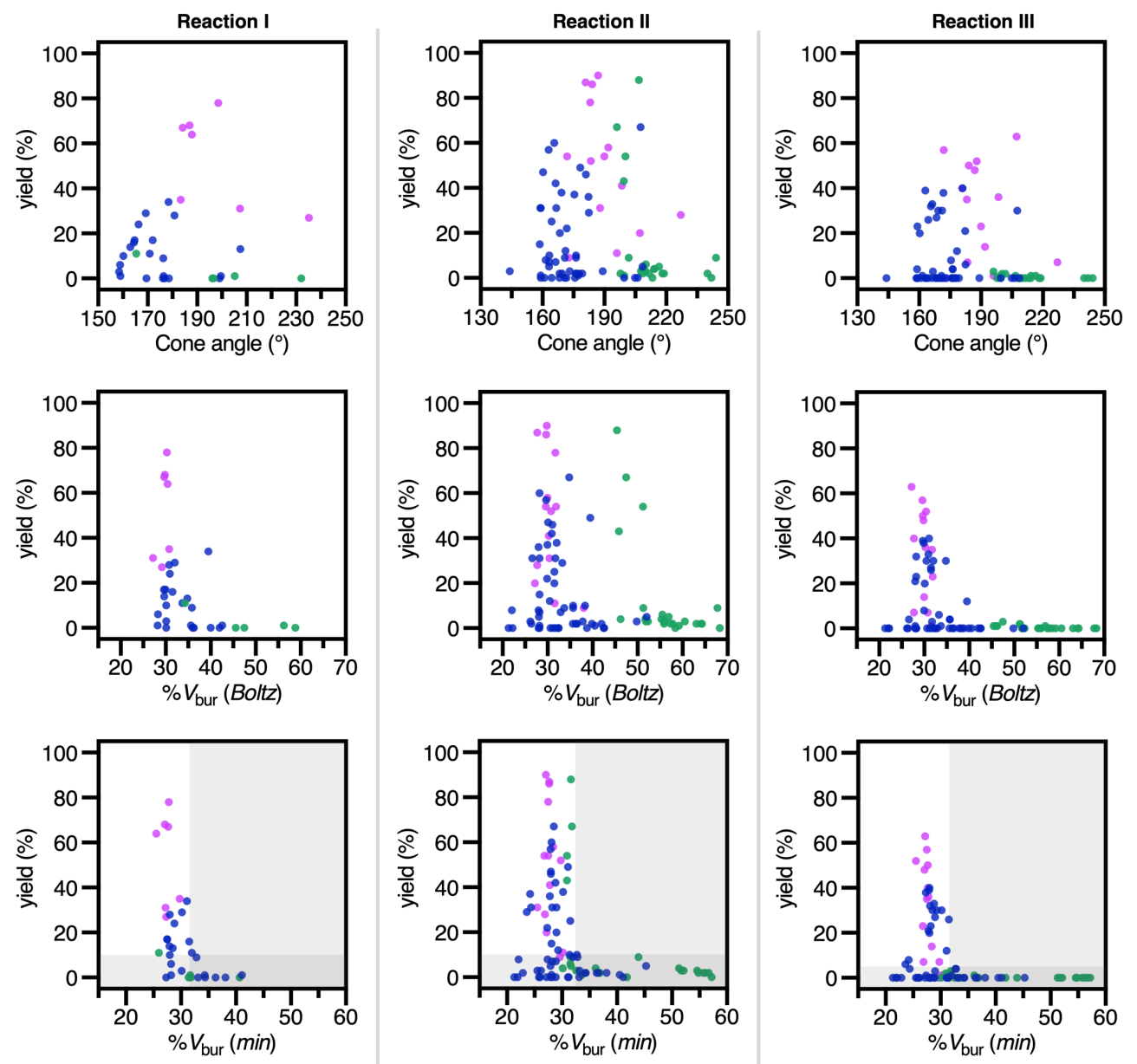

Fig. 2. Investigation of phosphine steric parameters in Ni catalyzed datasets. (A) Ni-Catalyzed SuzukiMiyaura datasets collected with monodentate phosphine ligands, including the DinoPhos ligands. (B) Analysis of datasets with steric parameters cone angle, $\% V_{\text {bur }}$ Boltz. and $\% V_{\text {bur }}$ min.. Pink dots $=$ DinoPhostype ligands, green dots $=$ Buchwald-type ligands, blue dots $=$ all others.

Mechanistic origin of $\% V_{\text {bur }}$ (min) reactivity thresholds. The presence of reaction-independent reactivity cliffs relative to $\% V_{\text {bur }}(\mathrm{min})$ in these data prompted us to investigate the mechanistic basis for this descriptor's significance. Given the importance of speciation in cross-coupling catalysis, we questioned whether this descriptor is predictive of the thermodynamic favorability of 
$128 \mathrm{~L}_{2} \mathrm{M}$ vs. $\mathrm{L}_{1} \mathrm{M}$ speciation. Historically, the development of large ligands - intuitively captured 129 through the cone angle descriptor - is an established principle to encourage $\mathrm{L}_{1} \mathrm{M}$ and achieve 130 reactivity in $\mathrm{Pd}$ catalysis (23-27). While TriceraPhos and TyrannoPhos both have cone angle 131 values higher than many phosphines known to form $\mathrm{L}_{1}$ species, $\% V_{\text {bur }}(\min )$ categorizes their 132 reactivity with those that form $\mathrm{L}_{2} \mathrm{Ni}$ and $\mathrm{Pd}$ complexes $(23,33)$. Thus, this interpretation of the 133 observed reactivity cliffs would suggest that steric bulk within the metal's first coordination sphere 134 primarily governs the ligation state outcome, regardless of the overall size of the phosphine.

135 To interrogate this hypothesis, we surveyed a subset (28 phosphines) of the ligands used across 136 the Ni-catalyzed $\mathrm{SMC}$ reactions to determine ligation state spectroscopically. $\mathrm{L}_{n} \mathrm{Ni}(4-$ 137 fluorobenzaldehyde) was chosen as a platform, as its three NMR handles $\left({ }^{1} \mathrm{H},{ }^{19} \mathrm{~F}\right.$, and $\left.{ }^{31} \mathrm{P}\right)$ 138 provided a readout of the in-situ ligation state at the metal center upon reaction of $\mathrm{Ni}(\mathrm{COD})_{2}$ with 139 the aldehyde and two equivalents of phosphine (VI, Figure 3A) (34, 35). Each ligand was assigned 140 as forming $\mathrm{L}_{2} \mathrm{Ni}$ or $\mathrm{L}_{1} \mathrm{Ni}$ complexes based on the observed spectra. The results of these experiments 141 were then evaluated with steric features from the library to determine which classified the ligands 142 into regions of similar ligation states (Figure 3B, see SI for additional parameters). As observed 143 with the cross-coupling reaction yields, cone angle was unable to partition the ligands. Indeed, the 144 DinoPhos ligands and DrewPhos all formed $\mathrm{L}_{2} \mathrm{Ni}$ complexes exclusively, despite having some of 145 the largest cone angle values among ligands evaluated. $\% V_{\text {bur }}($ Boltz) was able to correctly group 146 the DinoPhos-type ligands with other $\mathrm{L}_{2} \mathrm{Ni}$-forming phosphines; however, some flexible ligands 
147 (MeJohnPhos, $\mathrm{P}(i-\mathrm{Bu})_{3}$, and $\left.\mathrm{PBn}_{3}\right)$ remained

148 misclassified with $\mathrm{L}_{1} \mathrm{Ni}$-forming ligands.

149 Similar to the catalytic reactions, $\% V_{\text {bur }}(\mathrm{min})$ resolved these outliers and resulted in a sharp

matched the reactivity thresholds observed in

reactions I-V.

be challenging to access synthetically. To test

computed cone angle is $235^{\circ}$, one of the largest

angle value, its relatively low $\% V_{\text {bur }}(\mathrm{min})$ of only $27.2 \%$ suggested that PteroPhos should

this, we conceptualized a new DinoPhos ligand,

PteroPhos, which possesses two aryl groups

with

2,4,6-triisopropylphenyl

(TRIP)

substituents at the 3,5-positions (Figure 4A). Its
A. Spectroscopic determination of ligation state

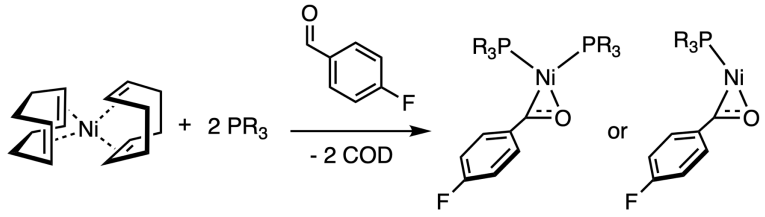

${ }^{1} \mathrm{H},{ }^{19} \mathrm{~F}, \&{ }^{31} \mathrm{P}$ NMR Detection

B. Analysis of experimental ligation state outcome
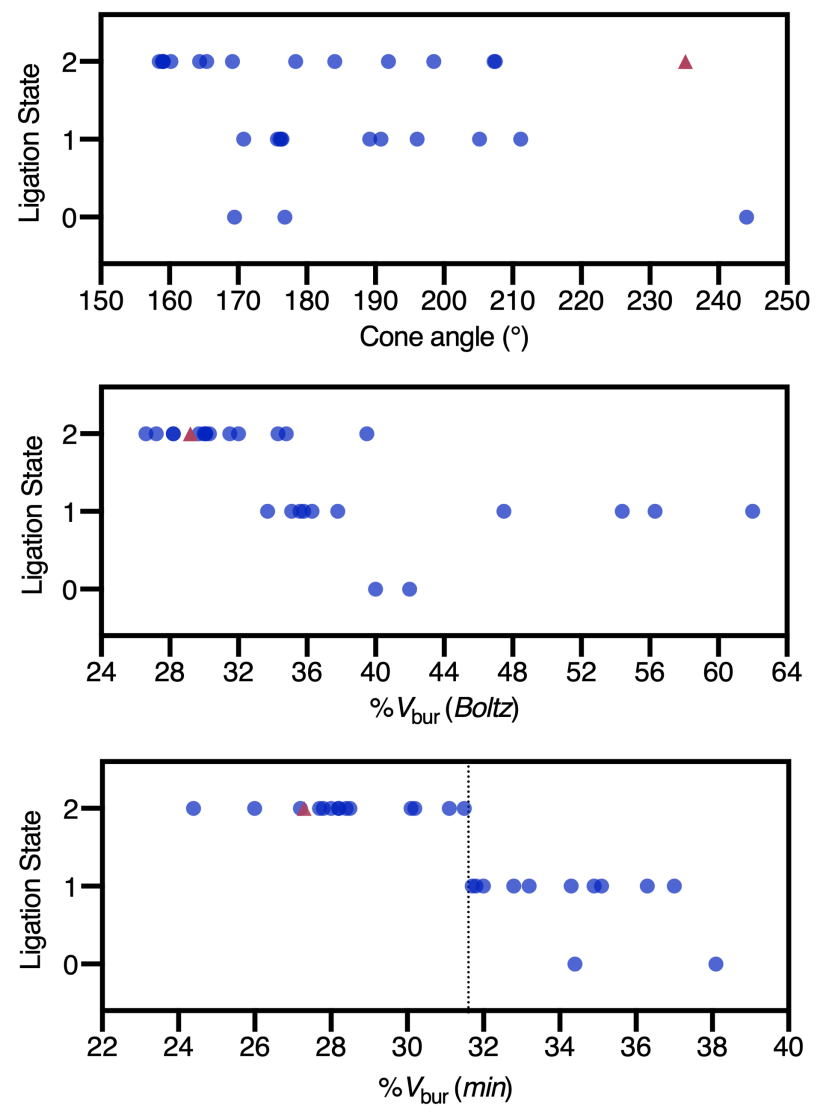

Fig. 3. Ligation state studies. (A) Reaction of $\mathrm{Ni}(\mathrm{COD})_{2}$, 4-fluorobenzaldehyde, and 2 equivalents of phosphine for spectroscopic determination of ligation state using ${ }^{1} \mathrm{H},{ }^{19} \mathrm{~F}$, and ${ }^{31} \mathrm{P} \quad \mathrm{NMR}$ spectroscopy in $\mathrm{C}_{6} \mathrm{D}_{6}\left(25^{\circ} \mathrm{C}\right)$. (B) Ligation state experiments plotted against cone angle, $\% V_{\text {bur }}$ (Boltz), and $\% V_{\text {bur }}(\mathrm{min})$. Red triangle represents PteroPhos. $(t-\mathrm{Bu})$ BrettPhos (non-ligating) datapoint beyond $\mathrm{x}$-axis range for $\% V_{\text {bur }}(\mathrm{min}) /($ Boltz) plots. 
170 ligand, we found that it readily formed a $\mathrm{L}_{2} \mathrm{Ni}$ (4-fluorobenzaldehyde) complex (Figure 3B) and 171 was moderately active in Ni-catalyzed reactions $\mathbf{I}-\mathbf{V}$, consistent with the $\% V_{\text {bur }}$ ( $\mathrm{min}$ ) classification 172 analysis.

173 We then sought to understand the structural significance of $\% V_{\text {bur }}$ ( $\min$ ) through 174 crystallographic and computational studies (complexes 1-4, Figure 4A), first to rationalize why 175 cone angle is not predictive of speciation. While our attempts at crystallizing $\mathrm{L}_{2} \mathrm{Ni}$ (benzaldehyde) 176 complexes bearing the DinoPhos ligands were not successful, we were able to obtain X-ray quality 177 crystals of (DrewPhos) ${ }_{2} \mathrm{Ni}(2$-naphthaldehyde) (3, Figure 4A). As observed in the solid state, the 178 two phosphines are positioned $116^{\circ}$ cis to each other on the complex; since DrewPhos possesses a 179 cone angle of $207^{\circ}$, a literal interpretation of cone angle as a description of phosphine size would 180 suggest that there is $90^{\circ}$ overlap of two solids, giving rise to considerable steric repulsion or 181 distortion. Yet, when compared with the X-ray structure of $\left(\mathrm{PPh}_{3}\right)_{2} \mathrm{Ni}$ (2-naphthaldehyde) (1, 182 Figure 4A), the two complexes exhibit very similar Ni-P bond lengths and $\mathrm{P}-\mathrm{Ni}-\mathrm{P}$ "bite angles", 183 despite DrewPhos having a cone angle $48^{\circ}$ larger than $\mathrm{PPh}_{3}$. What cone angle does not capture is 184 the non-uniform topology of the ligand structure distal to the metal. These less dense and more 185 conformationally flexible regions can be arranged in a manner that accommodates a second ligand 186 and/or substrate within the "cone", a recognized limitation that has led to the development of 187 alternative steric parameters $(16,36)$. On this basis, cone angle is unsuccessful at classifying the 188 ligation state and reactivity of the phosphines under study. Indeed, when compared with that of $L_{1}$ $189\left(\mathrm{P}(t-\mathrm{Bu})_{3}\right) \mathrm{Ni}$ (benzaldehyde) (2) (Figure 4A), the DFT-optimized structure of 190 (PteroPhos) ${ }_{2} \mathrm{Ni}$ (benzaldehyde) (4) (Figure 4A) provides the most striking example of $\mathrm{L}_{2}$ 191 complexation being agnostic to substantial remote steric bulk. 


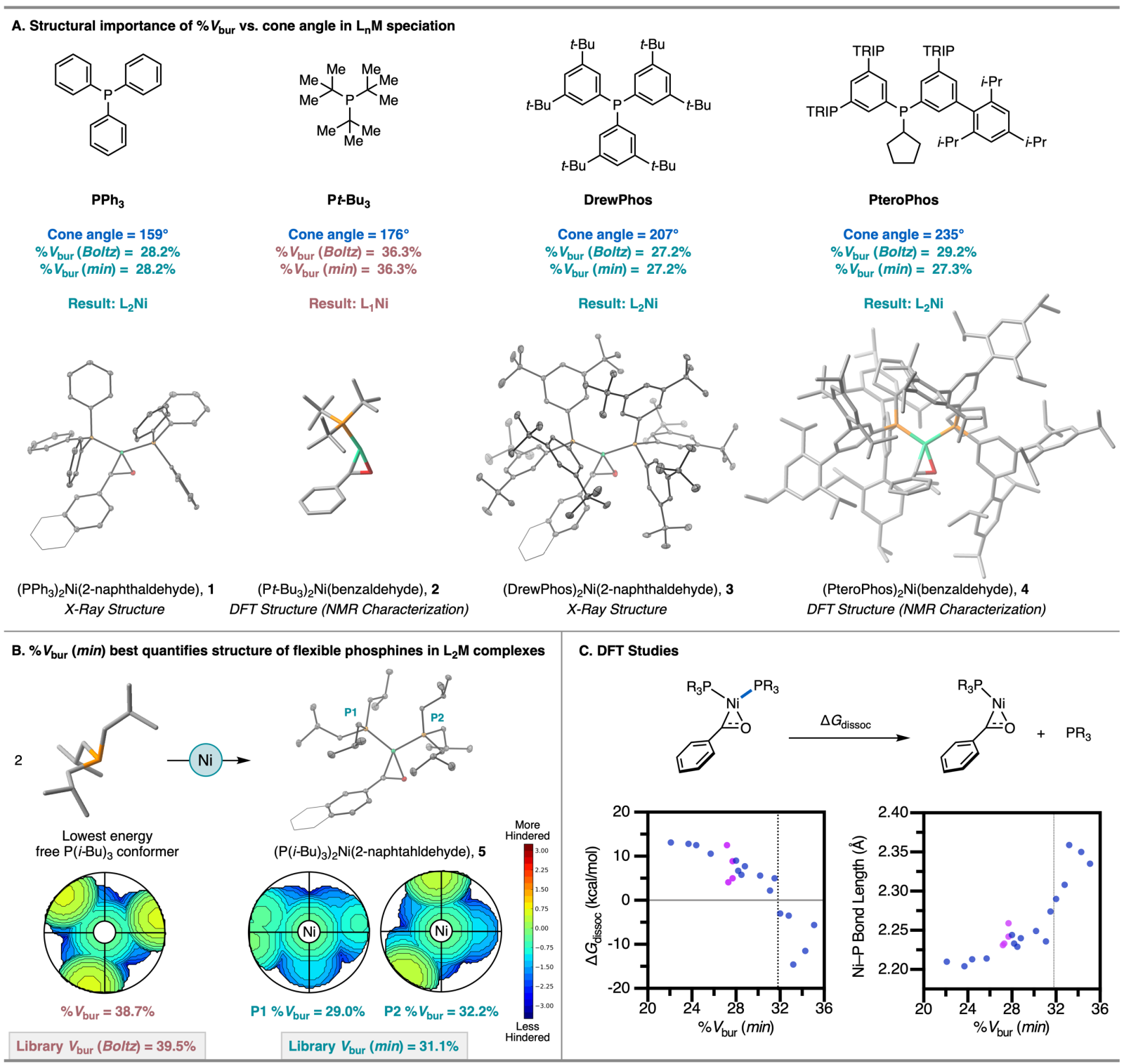

Fig. 4. Mechanistic studies of $\% V_{\text {bur }}$ (min) ligation state threshold. (A) Ligands with large cone angles but relatively small $\% V_{\text {bur }}$ values can form $\mathrm{L}_{2} \mathrm{Ni}$, supported structurally by solid-state structures of $\left(\mathrm{PPh}_{3}\right)_{2} \mathrm{Ni}$ (2-naphthaldehyde $)^{\mathrm{a}}$ and (DrewPhos) $)_{2} \mathrm{Ni}$ (2-naphthaldehyde) $)^{\mathrm{a}}$, along with DFT structures of $(\mathrm{P}(t$ $\left.\mathrm{Bu})_{3}\right) \mathrm{Ni}$ (benzaldehyde) and (PteroPhos) ${ }_{2} \mathrm{Ni}$ (benzaldehyde) optimized at the B3LYP-D3/6-31G(d,p)[SDD] level of theory. $(\mathbf{B})$ Formation of $\left(\mathrm{P}(i-\mathrm{Bu})_{3}\right)_{2} \mathrm{Ni}(2 \text {-naphthaldehyde })^{\mathrm{a}}$, with $\% V_{\text {bur }}$ values of the ligands bound are in agreement with the library value of $\% V_{\text {bur }}(\min )(\mathbf{C})$ DFT calculated dissociation energies and bond lengths for $\mathrm{L}_{2} \mathrm{Ni}$ (benzaldehyde) complexes. Ni-P bond length calculated from highlighted bond. DinoPhostype ligands (including DrewPhos) highlighted in purple. Calculations performed at M11-L/def2TZVP|SMD(benzene)//B3LYP-D3/6-31G(d,p)[SDD]|SMD(benzene) level of theory. ${ }^{\text {a }}$ (ORTEP diagram with $30 \%$ thermal ellipsoids shown. Hydrogens omitted for clarity).

While this case study highlights the dependence of speciation on steric bulk within the first coordination sphere, which is captured by the $\% V_{\text {bur }}$ parameters, the ligands investigated in Fig. 
4A had small variations between their $\% V_{\text {bur }}(\min )$ and $\% V_{\text {bur }}($ Boltz $)$ values. To better understand the structural importance of $\% V_{\text {bur }}(\mathrm{min})$ in the classification of the catalytic (Figure $2 \mathrm{~B}$ ) and ligation state experiments (Figure $3 \mathrm{~B}$ ), the $\mathrm{L}_{2} \mathrm{Ni}$-forming ligand with the greatest difference between $\% V_{\text {bur }}(\min )$ and $\% V_{\text {bur }}(B o l t z)$ values, $\mathrm{P}(i-\mathrm{Bu})_{3}$, was investigated. For this phosphine, lowest energy conformer and Boltzmann average $\% V_{\text {bur }}$ values $(38.7 \%$ and $39.5 \%$, respectively) are both substantially higher than other $\mathrm{L}_{2} \mathrm{Ni}$-forming ligands, and even higher than that of $\mathrm{L}_{1} \mathrm{Ni}$ forming $\mathrm{P}(t-\mathrm{Bu})_{3} \quad(36.3 \%)$. However, an $\mathrm{X}$-ray crystal structure of $\left(\mathrm{P}(i-\mathrm{Bu})_{3}\right)_{2} \mathrm{Ni}(2$ naphthaldehyde) (5) (Figure 4B) confirmed that two phosphines were bound to Ni, with $\% V_{\text {bur }}$ values determined to be $29.0 \%$ and $32.2 \%$, nearly matching that of $\% V_{\text {bur }}(\min )(31.1 \%)$. Thus, only $\% V_{\text {bur }}(\mathrm{min})$, which was designed to capture the degree of conformational flexibility, simulates the redistribution of steric bulk out of the metal's first coordination sphere in order to accommodate both ligands and the substrate for phosphines like $\mathrm{P}(i-\mathrm{Bu})_{3}$.

Finally, given the sharp catalytic and ligation state reactivity cliffs, we postulated that there would be a strong dependence on the thermodynamics of phosphine dissociation with $\% V_{\text {bur }}(\min )$. Out of all 28 phosphines studied in Figure 3, only one $\left(\mathrm{Cy}_{2} \mathrm{P}(t-\mathrm{Bu})\right)$ was found to have any spectroscopically observable equilibrium between $\mathrm{L}_{2} \mathrm{Ni}$ and $\mathrm{L}_{1} \mathrm{Ni}\left(1: 4 \mathrm{~L}_{2}: \mathrm{L}_{1}\right)$, with a $\% V_{\text {bur }}($ min) value of $32.0 \%$ near the speciation cutoff. Using $\mathrm{Cy}_{2} \mathrm{P}(t-\mathrm{Bu})$ as a reference, the free energy of ligand dissociation $\left(\Delta G_{\text {dissoc }}\right)$ was calculated for $20 \mathrm{~L}_{2} \mathrm{Ni}$ (benzaldehyde) complexes using DFT (Figure $4 \mathrm{C}$ ). A $\% V_{\text {bur }}(\min )$ cutoff of $32 \%$ cleanly separated the regions of positive and negative

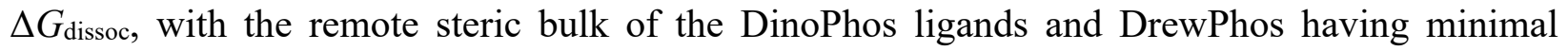
impacts on dissociation energy. Furthermore, the sharp downtick in the $\Delta G_{\text {dissoc }}$ values as the $\% V_{\text {bur }}$ (min) of the phosphine approached $32 \%$ corresponded with a significant uptick in the Ni-P bond length of the computed $\mathrm{L}_{2}$ complex, a reflection of the increased steric pressure caused by filling 
228 Ni's first coordination sphere at this value. Together, these studies demonstrate the strong physical 229 significance of the $\% V_{\text {bur }}(\mathrm{min})$ parameter and the $32 \%$ threshold value observed experimentally 230 in the datasets.

231 Development of threshold analysis algorithm. With the identification of sharp reactivity cliffs in

232 Ni SMC datasets, we sought to formalize the analysis to aid in the automated discovery of 233 thresholds and consequent classification of ligands within a diverse array of reaction datasets. 234 While $\% V_{\text {bur }}(\mathrm{min})$ was the most successful parameter in classifying ligands in the Ni-catalyzed 235 cross-coupling reactions, the workflow was designed to use all available parameters, and was 236 implemented as follows: the user first defines the \% yield or selectivity for a "successful" reaction 237 (either above "ligand-less" control reactivity or a single catalyst turnover), after which the data is 238 parsed by a single-node decision tree algorithm equipped with any descriptor sets provided to it. 239 The algorithm then determines the location of the threshold (if present) for each feature (see SI for 240 details). A consequence of this analysis is the dissection of datasets into four sectors of a confusion 241 matrix: 1) active ligands that meet the threshold speciation criterion (true positives), 2) inactive 242 ligands that correctly do not meet the criterion (true negatives), 3) active ligands that do not meet 243 the criterion (false negatives), and 4) inactive ligands that meet the criterion but fail for other 244 reasons (false positives) (37). These groupings can then in theory be analyzed further using 245 statistical modeling or as targets for mechanistic interrogation (38).

247 to computationally identify and quantify the thresholds observed empirically at the outset (see SI 248 for details). Most of the false positives in the classifications were ligands that were either electron249 poor or contained catalyst-poisoning functionality, and thus failed for reasons other than 
250

251

252

253

254

255

256

257

258

259

260

261

262

263

264

265

266

267

268

269

270

271

272

speciation. As an example, $\mathrm{P}\left(4-\mathrm{CF}_{3} \mathrm{Ph}\right)_{3}$ was found to form $\mathrm{L}_{2} \mathrm{Ni}$ (4-fluorobenzaldehyde) complexes but was inactive in all of the catalytic reactions.

Threshold analysis in Pd-catalyzed cross-coupling reactions. Considering the success of the classification tool for Ni-catalyzed cross-coupling reactions, we sought to determine if $\% V_{\text {bur }}(\min )$ could also be applied in identifying speciation more broadly. In particular, we investigated various

Pd-catalyzed cross-coupling reactions wherein the formation of $\mathrm{L}_{1} \mathrm{Pd}$ versus $\mathrm{L}_{2} \mathrm{Pd}$ species has been demonstrated to be a key facet of determining reaction outcome $(27,39)$. In one case study, we probed a Pd-catalyzed SMC of aryl triflates, for which $\mathrm{L}_{2} \mathrm{Pd}$ is proposed to be necessary for $\mathrm{C}-\mathrm{O}$ bond oxidative addition (VII, Figure 5A) $(27,40)$. The reliance on $\mathrm{L}_{2} \mathrm{Pd}$ in catalysis also provided an opportunity to test the DinoPhos ligands for the first time in Pd-catalysis. We generated a dataset using 56 phosphines and found a $\% V_{\text {bur }}(\mathrm{min})$ reactivity threshold at $32.4 \%$ (VII, Figure $5 \mathrm{~B}$ ). The reactivity cliff mirrored those of the $\mathrm{Ni}$ systems in both the cutoff value and directionality of active/inactive regions. Further, DrewPhos and CyTyrannoPhos were both correctly classified as active ligands based on their $\% V_{\text {bur }}(\mathrm{min})$ values below the threshold and performed similarly to "undecorated" $\mathrm{PPh}_{3}$ and $\mathrm{CyPPh}_{2}$, respectively. Spectroscopic studies corroborated these results, with the detection of $\mathrm{L}_{2} \mathrm{Pd}(\mathrm{dba})$ by ${ }^{31} \mathrm{P}$ NMR for DrewPhos and the DinoPhos ligands studied, including less active CyTriceraPhos (see SI for details). A necessity of the analysis for reaction VII was to partition and classify Buchwald-type phosphines separately, regardless of $\% V_{\text {bur }}(\min )$ (see SI). This is likely due to the fact that they can occupy two coordination sites, a design element of these ligands that allows them to mimic an $\mathrm{L}_{2} \mathrm{Pd}$ species during catalysis through stabilizing Pdarene interactions $(24,40,41)$. Consistent with previous investigations by the Sigman lab into the chemoselectivity of aryl triflate SMCs $(40), \mathrm{P}(o-\mathrm{Tol})_{3}$ was an outlier in the reaction, despite likely favoring $\mathrm{L}_{1} \mathrm{Pd}$ species under the reaction conditions (42). 
In the next set of case studies, various reactions where $\mathrm{L}_{1} \mathrm{Pd}$ species are implicated were

274 evaluated with the classification workflow. We generated datasets for two Pd-catalyzed SMC 275 couplings (VIII \& IX, Figure 5A) with aryl halides, including one reaction with sterically hindered 276 coupling partners, as well as a dataset for a Buchwald-Hartwig amination (X, Figure 5A). For the 277 two SMCs, the classification tool revealed a threshold at approximately $29 \% V_{\text {bur }}(\min )$, with the 278 active region occurring above that value, indicating that larger ligands - as defined by $\% V_{\text {bur }}$ 279 ( $\mathrm{min}$ - — are required for effective catalysis (VIII \& IX, Figure 5B). Notably, when comparing 280 reactions VII and VIII, the opposite directionalities of the observed thresholds are consistent with 281 the extensive literature precedent studying the effects of ligation state on the chemoselectivity of 282 aryl triflate vs. aryl chloride bond activation $(27,39,40)$. The presence of steric bulk on both 283 coupling partners in reaction IX did not significantly impact the value at which the threshold 284 occurred, analogous to what was observed for a sterically hindered nucleophile in reaction III of 285 the Ni-catalyzed datasets. Perhaps the most striking example is that of the Buchwald-Hartwig 286 amination (X, Figure 5B) with a clear reactivity cliff between high yielding reactions and those 287 with $0 \%$ yield at and below $31 \% V_{\text {bur }}(\mathrm{min})$. Two datasets mined from the literature were also 288 evaluated: a stereospecific Pd-catalyzed SMC previously studied by Biscoe, Sigman and 289 coworkers (18) (XI, Figure 5A) and a Heck reaction reported by Hartwig and coworkers (43) (XII, 290 Figure $5 \mathrm{~A}$ ). In both cases, $\% V_{\text {bur }}(\mathrm{min})$ thresholds were observed and suggest that $\mathrm{L}_{1} \mathrm{Pd}$ species are 291 responsible for catalysis (XI \& XII, Figure 5B). 

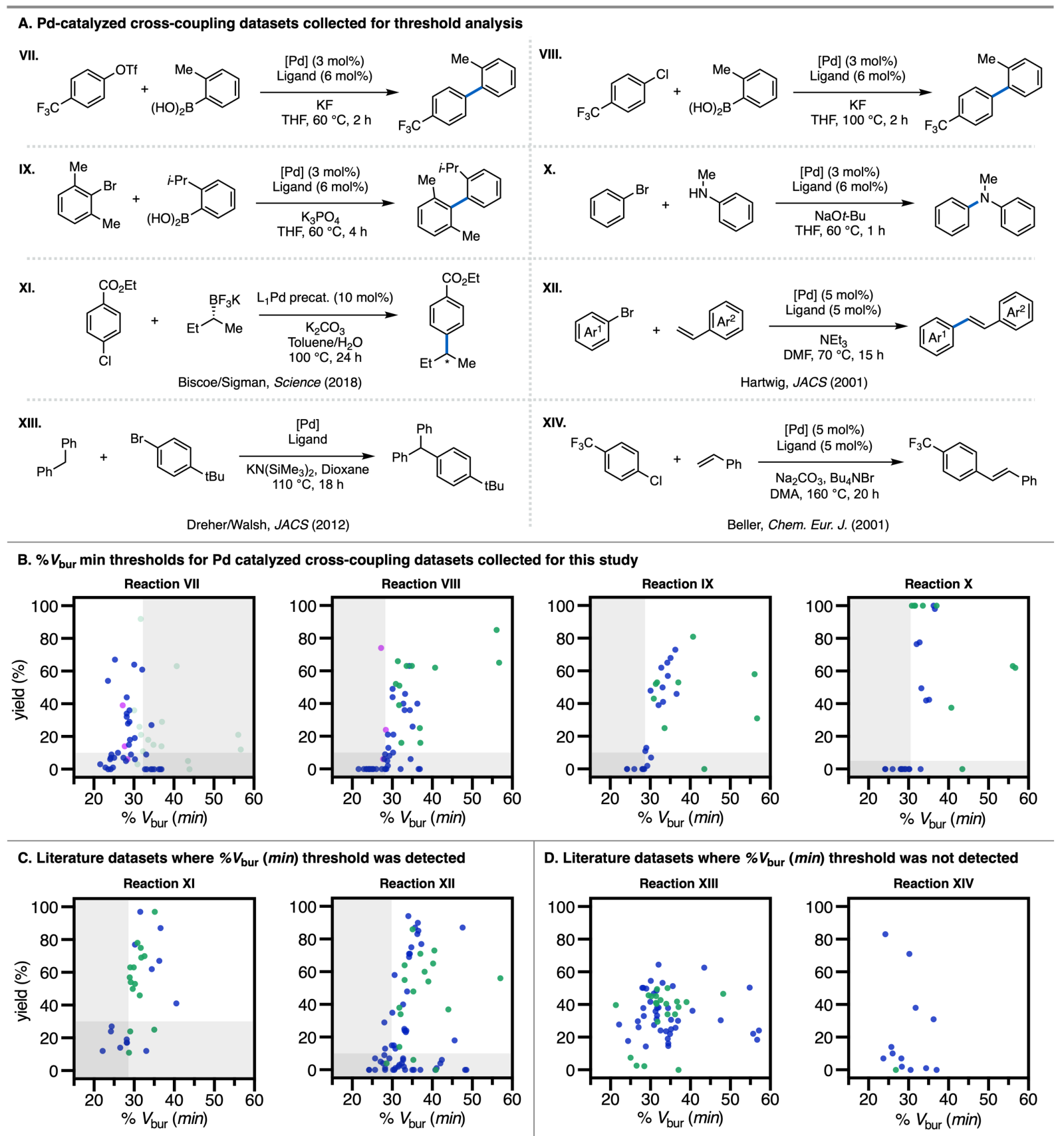

Fig. 5. Threshold analysis for Pd-catalyzed cross-coupling reactions. (A) Reaction schemes for reactions analyzed. VII-X collected for this study (see SI for exact reaction conditions), XI(18), XII (43), XIII(44), and XIV(45) mined from literature sources. (B) Threshold analysis of Pd datasets VII-X. (C) Threshold analysis of literature Pd datasets XI-XII. (D) Threshold analysis of literature Pd datasets XIII$\mathbf{X I V}$, where no $\% V_{\text {bur }}(\mathrm{min})$ threshold is present. For all plots, pink dots $=$ DinoPhos-type ligands, green dots $=$ Buchwald-type ligands, blue dots $=$ all others

We recognized that there are circumstances where ligand steric properties may not have as significant of an impact on the reaction outcome, and thus a threshold would not be expected. In 
our literature mining efforts, we identified two datasets where no phosphine steric threshold was found: a Pd-catalyzed $\mathrm{Csp}^{3}-\mathrm{H}$ arylation reported by Dreher, Walsh and coworkers (44) (XIII, Figure 5) and a Pd-catalyzed Heck cross-coupling studied by Beller and coworkers (45) (XIV, Figure 5). Possible explanations for these examples include high levels of ligandless background reactivity and/or catalytic cycles that are less sensitive to ligation state, possibly due to the generation of catalytically-active Pd nanoparticles $(46,47)$. The ability to rapidly identify these scenarios in datasets can serve as a valuable mechanistic probe given the physical importance of $\% V_{\text {bur }}(\min )$ (vide supra).

Summary and Outlook: The threshold values and directionality found for each of the datasets studied in this work are summarized in Fig. 6.

Comparing the $\mathrm{Ni}$ - and $\mathrm{Pd}$-catalyzed reactions reveals several mechanistically interesting features about $\mathrm{Ni}$ and $\mathrm{Pd}$ catalysis, as well as phosphine steric effects more generally. First, the opposite threshold directionalities of the Ni- and Pd-catalyzed Suzuki-Miyaura coupling reactions of aryl halides (II-V, IX, VIII) point to clear differences in mechanistic requirements between the two metals with regard to speciation for this reaction (48). Thus, the classification workflow provides a

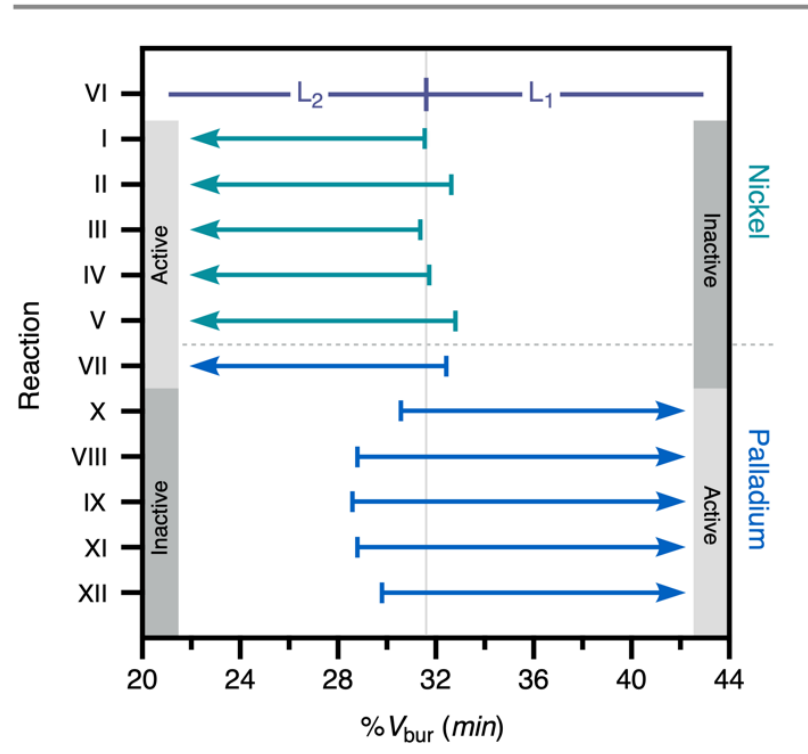

Fig. 6. Summary of reactivity thresholds. $\% V_{\text {bur }}$ ( $\mathrm{min})$ reactivity threshold locations and directionalities for all $\mathrm{Ni}$ (I-V (green)) and Pd (VIIXII (blue)) catalyzed reactions under study (excluding XIII \& VIV where no threshold was found). The $\mathrm{L}_{1}$ and $\mathrm{L}_{2}$ regions defined by the spectroscopic ligation state studies (VI (purple)). means to compare the two metals head-to-head and sheds light on the orthogonal ligand design principles often necessary for each metal $(19,49)$. Additionally, despite the differences in 
324 coordination sphere size between $\mathrm{Ni}$ and $\mathrm{Pd}$, the location of the threshold for the $\mathrm{Ni}$ datasets and 325 the Pd datasets with the sharpest reactivity cliffs (VII and X) occur at approximately the same 326 location of $32 \% V_{\text {bur }}(\mathrm{min})$, linking the catalytic outcome for both metals to ligand-controlled 327 thermodynamic $\mathrm{L}_{1}$ vs. $\mathrm{L}_{2}$ propensity. It is also worth noting that the observed thresholds occurred with different sensitivities and with some variation in value. Indeed, an overlap area can be observed wherein ligands with $\% V_{\text {bur }}$ ( $\min$ ) values between $29 \%$ and $32 \%$ work in all of the reactions tested with both metals (with the 331 exception of reaction $\mathbf{X})$. Accordingly, this may signify a region of $\% V_{\text {bur }}(\min )$ values in which $\mathrm{L}_{2} \mathrm{M}$ is thermodynamically favored in the resting state but $\mathrm{L}_{1} \mathrm{M}$ is still attainable; within this region, the equilibrium between $\mathrm{L}_{2} \mathrm{M}$ and $\mathrm{L}_{1} \mathrm{M}$ would be impacted by the temperature and concentration 334 of the reaction. This is known to be the case with phosphines such as $\mathrm{PCy}_{3}(39)$, and the decrease 335 in $\Delta G_{\text {dissoc }}$ between $29 \%$ and $32 \%$ (Figure 4 C) corroborates this hypothesis. Interestingly, even in 336 the data for Ni-catalyzed cross-couplings, the most active ligands generally fell within these limits 337 (Figure 2). This observation suggests that $\mathrm{L}_{1}$ intermediates and/or transition states may be relevant 338 in Ni catalysis $(50,51)$, but that the ability to attain an $\mathrm{L}_{2}$ resting state is necessary $(33,52,53)$, as 339 evidenced by the sharp reactivity cliffs. phosphine ligation state and reactivity in cross-coupling catalysis. In searching the feature space 342 of structurally diverse monodentate phosphines, we identified $\% V_{\text {bur }}(\min )$ as the parameter capable of bifurcating datasets on the basis of distinct mechanistic outcomes. We envision that this easily adopted tool should facilitate mechanistic studies of related organometallic reactions and 345 enable reaction development through prediction of active/inactive and mono/bis-ligating 346 phosphines prior to synthesis. While we recognize $\% V_{\text {bur }}(\mathrm{min})$ will not capture reactivity trends 
across all phosphines, the ability to identify outliers (especially false negatives) in the analysis can motivate the development of new parameters and targeted mechanistic study. Taken together, this study highlights how classification analysis can serve as an important mechanistic and predictive tool, complementary to LFERs, to facilitate statistical modeling of reactivity cliffs in chemistry.

\section{References:}

1. L. P. Hammett, The Effect of Structure upon the Reactions of Organic Compounds. Benzene Derivatives. Journal of the American Chemical Society 59, 96-103 (1937).

\section{P. R. Wells, Linear Free Energy Relationships. Chemical Reviews 63, 171-219 (1963).}

3. E. N. Jacobsen, W. Zhang, M. L. Guler, Electronic tuning of asymmetric catalysts. Journal of the American Chemical Society 113, 6703-6704 (1991).

\section{J. O. Schreck, Nonlinear Hammett relationships. J. Chem. Ed. 48, 103 (1971).}

5. C. B. Santiago, J. Y. Guo, M. S. Sigman, Predictive and mechanistic multivariate linear regression models for reaction development. Chem Sci 9, 2398-2412 (2018).

6. N. A. Romero, D. A. Nicewicz, Organic Photoredox Catalysis. Chemical Reviews 116, 10075-10166 (2016).

7. D. G. Brown, J. Boström, Analysis of Past and Present Synthetic Methodologies on Medicinal Chemistry: Where Have All the New Reactions Gone? J Med Chem 59, 4443-4458 (2016).

8. S. Xu, E. H. Kim, A. Wei, E.-i. Negishi, Pd- and Ni-catalyzed cross-coupling reactions in the synthesis of organic electronic materials. Science and Technology of Advanced Materials 15, 044201 (2014).

9. J. F. Hartwig, Organotransition Metal Chemistry: From Bonding to Catalysis. (University Science Books, 2010).

10. C. C. C. Johansson Seechurn, M. O. Kitching, T. J. Colacot, V. Snieckus, PalladiumCatalyzed Cross-Coupling: A Historical Contextual Perspective to the 2010 Nobel Prize. Angewandte Chemie International Edition 51, 5062-5085 (2012).

11. D. J. Durand, N. Fey, Computational Ligand Descriptors for Catalyst Design. Chem Rev 119, 6561-6594 (2019).

12. C. A. Tolman, Phosphorus ligand exchange equilibriums on zerovalent nickel. Dominant role for steric effects. J. Am. Chem. Soc. 92, 2956-2965 (1970). 
13. C. A. Tolman, Steric effects of phosphorus ligands in organometallic chemistry and homogeneous catalysis. Chem. Rev. 77, 313-348 (1977).

14. D. White, B. C. Tavener, P. G. L. Leach, N. J. Coville, Solid angles I. The radial profile. Journal of Organometallic Chemistry 478, 205-211 (1994).

15. K. C. Harper, E. N. Bess, M. S. Sigman, Multidimensional steric parameters in the analysis of asymmetric catalytic reactions. Nat Chem 4, 366-374 (2012).

16. A. Poater et al., SambVca: A Web Application for the Calculation of the Buried Volume of N-Heterocyclic Carbene Ligands. European Journal of Inorganic Chemistry 2009, 1759-1766 (2009).

17. H. Clavier, S. P. Nolan, Percent buried volume for phosphine and N-heterocyclic carbene ligands: steric properties in organometallic chemistry. Chem Commun (Camb) 46, 841-861 (2010).

18. S. Zhao et al., Enantiodivergent Pd-catalyzed $\mathrm{C}-\mathrm{C}$ bond formation enabled through ligand parameterization. Science 362, 670 (2018).

19. K. Wu, A. G. Doyle, Parameterization of phosphine ligands demonstrates enhancement of nickel catalysis via remote steric effects. Nat Chem 9, 779-784 (2017).

20. H. Y. Liu, K. Eriks, A. Prock, W. P. Giering, Quantitative analysis of ligand effects (QALE). Systematic study of iron-phosphorus bond lengths and their relationship to steric thresholds. Organometallics 9, 1758-1766 (1990).

21. T. Gensch et al., A comprehensive discovery platform for organophosphorus ligands for catalysis. ChemRxiv, (2021).

22. H. Clavier, S. P. Nolan, Percent buried volume for phosphine and N-heterocyclic carbene ligands: steric properties in organometallic chemistry. Chem. Comm. 46, 841-861 (2010).

23. F. Barrios-Landeros, B. P. Carrow, J. F. Hartwig, Effect of ligand steric properties and halide identity on the mechanism for oxidative addition of haloarenes to trialkylphosphine $\operatorname{Pd}(0)$ complexes. J Am Chem Soc 131, 8141-8154 (2009).

24. R. Martin, S. L. Buchwald, Palladium-Catalyzed Suzuki-Miyaura Cross-Coupling Reactions Employing Dialkylbiaryl Phosphine Ligands. Accounts of Chemical Research 41, 14611473 (2008).

25. A. A. Thomas, A. F. Zahrt, C. P. Delaney, S. E. Denmark, Elucidating the Role of the Boronic Esters in the Suzuki-Miyaura Reaction: Structural, Kinetic, and Computational Investigations. Journal of the American Chemical Society 140, 4401-4416 (2018).

26. G. C. Fu, The development of versatile methods for palladium-catalyzed coupling reactions of aryl electrophiles through the use of $\mathrm{P}(\mathrm{t}-\mathrm{Bu}) 3$ and $\mathrm{PCy} 3$ as ligands. Acc Chem Res 41, 15551564 (2008). 
27. F. Schoenebeck, K. N. Houk, Ligand-controlled regioselectivity in palladium-catalyzed cross coupling reactions. J Am Chem Soc 132, 2496-2497 (2010).

28. F.-S. Han, Transition-metal-catalyzed Suzuki-Miyaura cross-coupling reactions: a remarkable advance from palladium to nickel catalysts. Chemical Society Reviews 42, 5270-5298 (2013).

29. A. P. Cinderella, B. Vulovic, D. A. Watson, Palladium-Catalyzed Cross-Coupling of Silyl Electrophiles with Alkylzinc Halides: A Silyl-Negishi Reaction. J Am Chem Soc 139, 7741-7744 (2017).

30. Cone angle values used in text are those for the Boltzmann weighted average of the conformational ensemble from the descriptor library. Minimum cone angles were also evaluated but for simplicity are shown in the SI.

31. A. J. Kendall, L. N. Zakharov, D. R. Tyler, Steric and Electronic Influences of BuchwaldType Alkyl-JohnPhos Ligands. Inorg Chem 55, 3079-3090 (2016).

32. Buchwald-type phosphines are known to act as structurally bidentate-like ligands with $\mathrm{Pd}$ due to stabilizing interactions between the arene and the metal. While little is currently known about the structural behavior of these ligands with $\mathrm{Ni}$, this semi-bidentate binding may enable certain Ni-catalyzed reactions to occur despite only one phosphine bound; their mode of action in these case studies is the subject of ongoing investigation..

33. E. A. Standley, S. J. Smith, P. Müller, T. F. Jamison, A Broadly Applicable Strategy for Entry into Homogeneous Nickel(0) Catalysts from Air-Stable Nickel(II) Complexes. Organometallics 33, 2012-2018 (2014).

34. D. Walther, Reaktionen von heteroolefinen an zentralmetallen in niedrigen oxidationsstufen: stabile aldehydkomplexe des nickel $(\mathrm{O})$ und verwandte verbindungen. Journal of Organometallic Chemistry 190, 393-401 (1980).

35. M. Ohashi, H. Saijo, T. Arai, S. Ogoshi, Nickel(0)-Catalyzed Formation of Oxaaluminacyclopentenes via an Oxanickelacyclopentene Key Intermediate: Me2AlOTf-Assisted Oxidative Cyclization of an Aldehyde and an Alkyne with Nickel(0). Organometallics 29, 65346540 (2010).

36. I. A. Guzei, M. Wendt, An improved method for the computation of ligand steric effects based on solid angles. Dalton Transactions, 3991-3999 (2006).

37. Given that other ligand features can and will lead to poor catalyst performance, the presence of false positives should not be viewed as detrimental to the success/accuracy of the classifier.

38. Z. L. Niemeyer et al., Parameterization of Acyclic Diaminocarbene Ligands Applied to a Gold(I)-Catalyzed Enantioselective Tandem Rearrangement/Cyclization. Journal of the American Chemical Society 139, 12943-12946 (2017). 
39. A. F. Littke, C. Dai, G. C. Fu, Versatile Catalysts for the Suzuki Cross-Coupling of Arylboronic Acids with Aryl and Vinyl Halides and Triflates under Mild Conditions. Journal of the American Chemical Society 122, 4020-4028 (2000).

40. Z. L. Niemeyer, A. Milo, D. P. Hickey, M. S. Sigman, Parameterization of phosphine ligands reveals mechanistic pathways and predicts reaction outcomes. Nat Chem 8, 610-617 (2016).

41. K. L. Billingsley, K. W. Anderson, S. L. Buchwald, A Highly Active Catalyst for SuzukiMiyaura Cross-Coupling Reactions of Heteroaryl Compounds. Angewandte Chemie International Edition 45, 3484-3488 (2006).

42. J. F. Hartwig, F. Paul, Oxidative Addition of Aryl Bromide after Dissociation of Phosphine from a Two-Coordinate Palladium(0) Complex, Bis(tri-o-tolylphosphine)Palladium(0). Journal of the American Chemical Society 117, 5373-5374 (1995).

43. J. P. Stambuli, S. R. Stauffer, K. H. Shaughnessy, J. F. Hartwig, Screening of Homogeneous Catalysts by Fluorescence Resonance Energy Transfer. Identification of Catalysts for Room-Temperature Heck Reactions. Journal of the American Chemical Society 123, 26772678 (2001).

44. J. Zhang, A. Bellomo, A. D. Creamer, S. D. Dreher, P. J. Walsh, Palladium-Catalyzed C(sp3)-H Arylation of Diarylmethanes at Room Temperature: Synthesis of Triarylmethanes via Deprotonative-Cross-Coupling Processes. Journal of the American Chemical Society 134, 1376513772 (2012).

45. A. Zapf, M. Beller, Palladium Catalyst Systems for Cross-Coupling Reactions of Aryl Chlorides and Olefins. Chemistry - A European Journal 7, 2908-2915 (2001).

46. S. S. Zalesskiy, V. P. Ananikov, Pd2(dba)3 as a Precursor of Soluble Metal Complexes and Nanoparticles: Determination of Palladium Active Species for Catalysis and Synthesis. Organometallics 31, 2302-2309 (2012).

47. N. W. J. Scott et al., The ubiquitous cross-coupling catalyst system 'Pd(OAc)2'/2PPh3 forms a unique dinuclear PdI complex: an important entry point into catalytically competent cyclic Pd3 clusters. Chemical Science 10, 7898-7906 (2019).

48. S. Z. Tasker, E. A. Standley, T. F. Jamison, Recent advances in homogeneous nickel catalysis. Nature 509, 299-309 (2014).

49. C. M. Lavoie, M. Stradiotto, Bisphosphines: A Prominent Ancillary Ligand Class for Application in Nickel-Catalyzed C-N Cross-Coupling. ACS Catalysis 8, 7228-7250 (2018).

50. H. Shirataki, M. Ohashi, S. Ogoshi, Nickel-Catalyzed Three-Component Coupling Reaction of Tetrafluoroethylene and Aldehydes with Silanes via Oxa-Nickelacycles. European Journal of Organic Chemistry 2019, 1883-1887 (2019). 
482 51. We recognize that there are some instances where $\mathrm{Ni}$ will be $\mathrm{L}_{1}$ throughout the catalytic 483 cycle allowing ligands like $\mathrm{P}(t-\mathrm{Bu})_{3}$ to be employed successfully. See ref (50).

484 52. N. Hazari, P. R. Melvin, M. M. Beromi, Well-defined nickel and palladium precatalysts 485 for cross-coupling. Nat Rev Chem 1, (2017).

486 53. P.-A. Payard, L. A. Perego, I. Ciofini, L. Grimaud, Taming Nickel-Catalyzed Suzuki487 Miyaura Coupling: A Mechanistic Focus on Boron-to-Nickel Transmetalation. ACS Catalysis 8, 488 4812-4823 (2018).

489

490

Acknowledgements: The authors thank Dr. Phil Jeffrey for assistance with X-Ray structure

491 determination and Dr. Kevin Wu for helpful discussions and directions. Additionally, we thank

492 Prof. F. Dean Toste for helpful discussions related to the preparation of the manuscript.

493 Funding: NSF CCI Center for Computer Assisted Synthesis (CHE-1925607)

494 Author Contributions: T.G., M.S.S., and A.G.D. conceived the project. S.H.N.-S., S.R.S., J.E.B., 495 and H.C.J. performed the experiments and analyzed the data. E.P., T.G., and S.R.S. developed the 496 classification algorithm. M.S.S. and A.G.D. oversaw the project. S.H.N.-S., J.E.B., S.R.S., M.S.S., 497 and A.G.D. wrote the manuscript with contributions from all authors.

498 Competing Interests: The authors declare no competing interests.

499 Data and Materials Availability: X-ray crystallographic data are available free of charge from 500 the Cambridge Crystallographic Data Centre, under reference numbers 2069892-2069895. 501 Coordinates of all computed structures are included in a separate zip file. All other experimental, 502 computational, spectroscopic, and crystallographic data are included in the supplementary 503 materials. The code used in this project is available at https://github.com/SigmanGroup/Threshold.

504 Supplementary Materials

505 Materials and Methods

$506 \quad$ Figs. S1 to S116

507 Tables S1 to S18

508 References

509 